\title{
DISEÑO DE UN SISTEMA EXPERTO PARA REUTILIZACIÓN DE AGUAS RESIDUALES TRATADAS
}

\section{DESIGN OF AN EXPERT SYSTEM FOR REUSING TREATED WASTEWATER}

\author{
María Camila Escobar', Luis Felipe Tovar², Jonathan Romero Cuéllar ${ }^{3}$
}

Fecha de recepción: 7 de abril de 2016

Fecha de revisión: 13 de mayo de 2016

Fecha de aprobación: 20 de junio de 2016

Referencia: M. C. Escobar, L. F. Tovar, J. Romero Cuéllar (2016). Diseño de un sistema experto para reutilización de aguas residuales tratadas. Ciencia e Ingeniería Neogranadina, 26 (2), pp. 21-34, DOI: http://dx.doi.org/10.18359/rcin. 1827

\begin{abstract}
RESUMEN
Este trabajo propone un sistema experto basado en lógica difusa para apoyar la toma de decisiones en proyectos de reutilización de aguas residuales domésticas tratadas. La herramienta hidroinformática diseñada estima el potencial de reutilización de agua residual tratada a partir de factores como el índice de escasez y la eficiencia en $\mathrm{DBO}_{5}$ de una planta de tratamiento de aguas residuales (PTAR). La herramienta fue validada a través de un caso de estudio en la PTAR del municipio de Nátaga, Huila, Colombia. En el caso de estudio, se utilizó el balance hídrico, análisis de calidad del agua y encuestas a expertos para establecer la estructura del sistema de lógica difusa, el cual fue implementado en Matlab ${ }^{\circledast}$. Los resultados indican una eficiencia del tratamiento de $88,7 \%$, un índice de escasez de 28,5, que corresponde a un índice de escasez medio alto, y por tanto el sistema experto recomienda la reutilización de agua residual tratada con un $92,2 \%$, lo que se clasifica como una reutilización confiable justificada. Se concluye que el sistema experto es adecuado para identificar fuentes alternas de recursos hídricos y esta información puede ayudar a los tomadores de decisiones en la implementación de políticas de gestión integral del recurso hídrico.
\end{abstract}

1. Ing. Agrícola, joven investigadora, Universidad Surcolombina, Neiva, Colombia, u2008276613@usco.edu.co

2. Ing. Agrícola, joven investigador, Universidad Surcolombina, Neiva, Colombia, u2008171952@usco.edu.co

3. Ing. Agrícola, MSc. Hidrosistemas, investigador, Centro de Investigación en Ciencias y Recursos GeoAgroAmbientales - Cenigaa, Neiva, Colombia, jonathan.romero@cenigaa.org 
Palabras clave: lógica difusa, sistema de soporte de toma de decisiones, recursos hídricos, potencial de reutilización de aguas, PTAR, clima tropical.

\section{ABSTRACT}

This work describes an expert system that uses fuzzy logic to back decision support systems (DSS) up for a municipal treated wastewater reuse project. The hydroinformatic tool calculates water reuse potential. This tool uses two parameters: a drought index and BOD $\mathrm{D}_{5}$ wastewater treatment rate. The DSS was validated in the municipal wastewater treatment plant (MWTP) of Nátaga, Huila, Colombia. The methodology used water balance, water quality analysis, and expert surveys. The fuzzy logic toolbox of Matlab (MathWorks) was used to implement the fuzzy inference system (FIS). The results showed a wastewater treatment rate of $88.7 \%$ and drought index of 28.5, which means a high-medium drought index. Therefore, the expert system suggests water reuse of $92.2 \%$. The output of systems is classified as a reliable and justified water reuse. It is concluded that the expert system is suitable to identify alternative sources of water resources. Finally, the decision support tools can help governments to implement policies that integrate management of water resources.

Keywords: fuzzy logic, decision support System (DSS), water resources, potential water reuse, WWTP, tropical climate.

\section{INTRODUCCIÓN}

A medida que las sociedades se desarrollan enfrentan problemas cada vez más complejos; en ese sentido, surge la necesidad de tomar decisiones de manera informada, basadas en conocimiento, contexto local y consenso social [1]. Especificamente en el campo ambiental y de acuerdo con la propuesta de [2] se plantean sistemas de soporte de decisiones ambientales como estrategia para tomar decisiones con un menor grado de incertidumbre. De igual forma, [3] plantea la necesidad de identificar los factores de riesgo en la seguridad hídrica para desarrollar un sistema de toma de decisiones que propenda por un manejo eficiente del recurso hídrico. Un ejemplo de problemas complejos son los proyectos de reutilización de aguas residuales tratadas porque tienen en cuenta muchas variables que interactúan entre sí, a saber: ambientales, jurídicas, económicas, sociales, políticas, institucionales y culturales [4].

En los proyectos de reutilización de aguas residuales tratadas para riego agrícola no solo se analiza la calidad del agua residual sino también las características y necesidades del cultivo y suelo. Estudios como los de [4-5] proponen el siguiente esquema de trabajo: para la caracterización de la zona de estudio se tienen en cuenta topografía, clima, suelo y el cultivo (limitaciones, potencialidades y requerimientos). Desde el punto de vista de la calidad del agua para riego se debe hacer la caracterización físico-química del efluente, de acuerdo con las directrices de la FAO (1999) [6], y la caracterización microbiológica, de acuerdo con las directrices de la OMS (1989) [7], evaluando CF y huevos de helmintos $(\mathrm{HH})$. 
Finalmente, para la eficiencia de la PTAR, se caracterizan el afluente y el efluente con la demanda bioquímica de oxígeno (DBO), los sólidos suspendidos (SS), N, P, K, CF y HH. Todos estos requerimientos deben estar de acuerdo con la normatividad vigente de cada país.

En términos de normas sobre reutilización de aguas residuales tratadas para riego agrícola, Colombia acoge las normas internacionales [6-7]. A nivel nacional el Decreto 3930 de 2010 [8] define y promueve el reúso de aguas residuales a través de los planes de reconversión a tecnologías limpias en gestión de vertimientos y lo incluye en la gradualidad para cumplimiento.

En Colombia el problema de la reutilización es el bajo porcentaje de aguas residuales tratadas que generan riesgos en la salud pública [9]. Colombia tiene una superficie irrigada con aguas residuales de 1'230.193 ha, con $27 \%$ de agua residual tratada y $73 \%$ sin tratar, por lo general diluida con aguas superficiales [10]. Por otro lado, se afirma que el número de plantas de tratamiento no es lo sustancial, el problema es la falta de un óptimo mantenimiento y buen uso de la infraestructura [11]. Una posible alternativa sería la reutilización en cultivos no comestibles como [12].

Los proyectos de reutilización de aguas residuales tratadas actualmente cobran especial interés porque son la única forma de ampliar la oferta hídrica de un ecosistema y una alternativa para evitar la contaminanción de las fuentes hídricas [13]. Estos proyectos son muy útiles, especialmente durante periodos de estiaje prolongados por efectos de fenómenos de variabilidad climática extrema o cambio climático. En este sentido, los proyectos de reutilización de agua residual son considerados como medidas de adaptación al cambio climático que promueven una gestión ambiental más sostenible.

De acuerdo con el Estudio Nacional del Agua (ENA) 2014 [11], los eventos secos en Colombia tienen mayor extensión espacial. De igual forma, la extensión temporal de los periodos de sequía son alrededor de 2 meses para la cuenca Magdalena-Cauca. Por otro lado, a nivel regional se evidencia la pérdida del rendimiento y la regulación hídrica de la cuenca hidrográfica del Alto Magdalena. De hecho, el $86 \%$ de los municipios del Huila presentan serios problemas en el suministro de agua potable [14]. En este sentido es necesario identificar fuentes adicionales para satisfacer las demandas del recurso hídrico. Recientemente una nueva variable agrava la problemática: el cambio climático.

El calentamiento global, el crecimiento poblacional, el cambio climático y los fenómenos de variabilidad climática extrema generan un riesgo para la sustentabilidad del recurso hídrico [15]. A nivel regional y de acuerdo con las proyecciones de la Segunda Comunicación de Cambio Climático del Ideam, el norte del Huila tendrá una disminución del $30 \%$ en la precipitación. En otras palabras, y de acuerdo con los escenarios de cambio climático formulados por el Panel Intergubernamental de Cambio Climático (IPCC) (escenario A2), el Huila será más seco de lo que es hoy día [16].

Por otro lado, y de acuerdo con [17-18], los sistemas inteligentes son técnicas computacionales capaces de modelar y analizar problemas muy complejos. Para este tipo de problemas las técnicas convencionales no son realmente soluciones costo-eficientes. Por tanto, en lasúltimas tres décadas, investigadores han desarrollado interesantes aplicaciones de sistemas inteligentes para resolver problemas 
complejos en el campo ambiental y agrícola. Un ejemplo de estos desarrollos es la lógica difusa. Dentro de las principales aplicaciones se encontró: modelamiento y predicción (24\%), control $(28 \%)$, clasificación $(24 \%)$, agrupación difusa $(9 \%)$, inferencia a base de reglas $(7 \%)$, fusión de datos multisensores (4\%), optimización $(1 \%)$ y otros $(3 \%)$.

Además, autores como [19-21] proponen sistemas de toma de decisiones multicriterio para resolver problemas de gestión del recurso hídrico basados en sistemas inteligentes. En Colombia se han implementado metodologías para el diagnóstico del saneamiento en comunidades de desplazados en situación de emergencia [22], teoría de conjuntos difusos aplicada al estudio de la dinámica del agua y de los solutos en el suelo [23], los sistemas difusos como herramienta de modelación de la producción frutícola [24], entre otras.

De acuerdo con lo anterior, esta investigación consiste en diseñar un sistema experto basado en lógica difusa para apoyar la toma de decisiones en proyectos de reutilización de aguas residuales domésticas tratadas. La herramienta hidroinformática diseñada estima el potencial de reutilización de agua residual tratada a partir de factores como el índice de escasez y la eficiencia de la planta de tratamiento de agua residual (PTAR). La herramienta fue validada a través de un caso de estudio en la PTAR del municipio de Nátaga.

\section{METOdOLOGÍA}

\subsection{Zona de estudio}

El municipio de Nátaga está localizado en la región suroccidental del departamento del Huila, su cabecera municipal se encuentra a $2^{\circ}$ y $34^{\prime \prime}$ de latitud norte y $75^{\circ} 49^{\prime \prime}$ de longitud oeste; a una distancia de 120 km del municipio de Neiva, capital del departamento. Tiene una superficie de $132,87 \mathrm{~km}^{2}$, que equivalen a $13.286,88$ hectáreas, de las cuales solamente 44,6 pertenecen al perímetro urbano actual. Este municipio comprende temperaturas que van desde los $12{ }^{\circ} \mathrm{C}$ hasta $24^{\circ} \mathrm{C}$, con una altura al nivel del mar de 1.480 m [25].

\subsection{Balance hídrico}

Se aplicó el método del balance hídrico [26] para establecer las condiciones hidroclimatológicas de la zona de estudio. Los datos climatológicos fueron tomados de la estación climatológica Nátaga (21055020), la cual es administrada por el Ideam (Instituto de Hidrología, Meteorología y Estudios Ambientales). Especificamente, se utilizaron las variables precipitación y evapotranspiración potencial mensual multianual desde 1993 hasta 2013.

\subsection{Caracterización de la PTAR}

La PTAR del municipio de Nátaga se encuentra ubicada a $2^{\circ} 32^{\prime} 28^{\prime \prime}$ latitud norte y $75^{\circ} 48^{\prime} 38.2^{\prime \prime}$ longitud oeste. A continuación se describe el esquema de tratamiento actual de la PTAR (sin la propuesta de reutilización de agua residual). La PTAR cuenta con un tratamiento preliminar y secundario para el mejoramiento de la calidad del agua residual del centro poblado. La PTAR es de tipo anaeróbico y se compone de un tratamiento preliminar que consta de dos rejillas de cribado para la remoción de material en suspensión, dos desarenadores en paralelo con el fin de depositar el material de sedimentos y una canaleta Parshall para el monitoreo del caudal entrante a la planta. 
El tratamiento secundario está conformado por cuatro UASB (Upflow anaerobic sludge blanket); en estos reactores se lleva a cabo el proceso de degradación anaerobia. Los productos finales de la digestión anaerobia son: agua, material celular y biogás. Este último está generalmente constituido por metano $(70 \%)$, bióxido de carbono $(29,5 \%)$ y trazas de ácido sulfhídrico y nitrógeno (0,5\%). También se compone de dos filtros anaerobios de flujo ascendente (FAFA). Luego de este tratamiento se pasa a cuatro lechos de secado para, finalmente, verter el efluente tratado a la quebrada El Pueblo.

\subsection{Diseño del sistema de lógica difusa}

Los proyectos de reutilización de aguas residuales tratadas son de mucha complejidad debido a la gran cantidad de variables y sus posibles interrelaciones. Por ejemplo, inicialmente se tiene en cuenta una condición de salud pública (contaminación microbiológica), implicaciones económicas, sociales, ambientales, jurídicas, usos del agua y productivas. En este sentido y teniendo en cuenta la gran cantidad de posibles relaciones que se pueden establecer entre las variables del sistema, se buscó una herramienta metodológica que ayuda a tomar decisiones de manera acertada teniendo en cuenta la información disponible, las variables del problema y las posibles soluciones. Por tanto, luego de una evaluación preliminar y una revisión de literatura, se seleccionó la lógica difusa como herramienta para abordar el problema.

\subsubsection{Lógica difusa}

Para afrontar el problema se elaboró un sistema de lógica difusa tipo 'Mamdani', que consta de un motor de inferencia, una base de conocimiento y una interfaz, tanto de fuzificación como de defuzificación, para procesar las entradas y salidas del sistema (Figura 1). Para construir la interfaz gráfica de usuario se utilizó la caja de herramientas de lógica difusa del software Matlab.

Los sistemas basados en lógica difusa tienen la ventaja de convertir variables cualitativas en cuantitativas a través de conjuntos difusos, operadores lógicos y manipulaciones matemáticas. Esta característica es de mucha utilidad cuando se trabaja con conocimiento experto.

Los sistemas de lógica difusa usan modelos de razonamiento (Si-Entonces) para aproximar el razonamiento humano y manejar la incertidumbre [28]. Para los valores de entrada

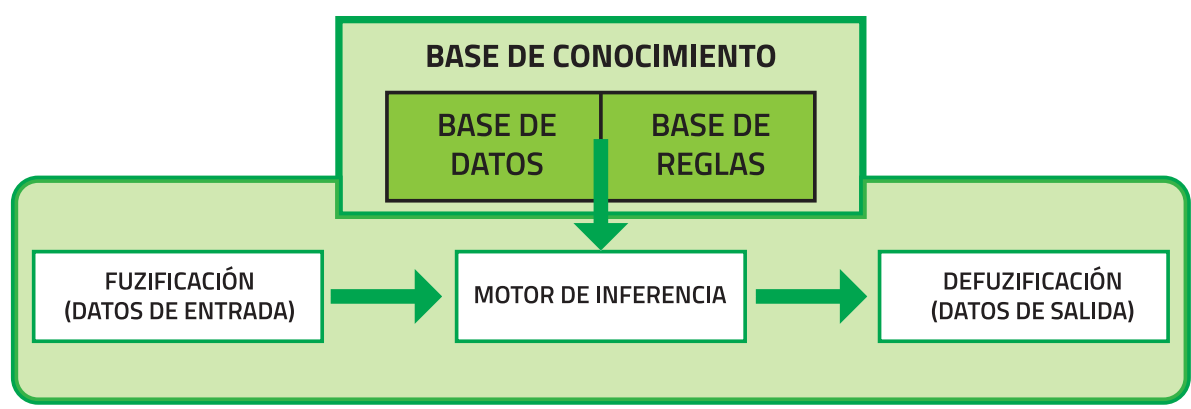

Figura 1. Estructura de un modelo difuso.

Fuente: Salazar (2012) [27]. 
y salida en la interfaz difusa se asignaron a cada una de las variables lingüísticas una función de membresía con un dominio de 0 a 1, la cual, al ser evaluada con el valor de entrada de la variable, nos permite saber qué "tan cierto" es que pertenezca al término lingüístico asociado a dicha función. Para este caso se seleccionó una función tipo triangular.

\subsubsection{Identificación de las variables del sistema}

Dentro del marco de posibilidades las variables del sistema son muchas, por ejemplo: demanda, oferta, calidad del agua, usos del agua, eficiencias de tratamiento, reglamentación vigente, condiciones culturales, balance hídrico, disponibilidad de información, calidad y validez de la información, etc. Finalmente, y luego de un comité de expertos, se concluyó que las variables de entrada que más pueden aportan información a la toma de decisiones fueron: la oferta y demanda hídrica, la eficiencia de tratamiento en $\mathrm{DBO}_{5}$ como variables de entrada y la reutilización del agua como variable de salida. La oferta y demanda hídrica se representa a través del índice de escasez.

Índice de escasez: para el cálculo de dicha variable se tendrá en cuenta la oferta y demanda hídrica de la zona de estudio, según la Resolución 0865 del 22 de julio de 2004, por la cual se adopta la metodología para el cálculo del Índice de escasez para aguas superficiales (Tabla 1), desarrollada por el Instituto de Hidrología, Meteorología y Estudios Ambientales (Ideam), definiéndose este como la relación porcentual entre la demanda de agua del conjunto de actividades sociales y económicas con la oferta hídrica disponible, luego de aplicar factores de reducción por calidad del agua y caudal ecológico.

El Índice de escasez representa la relación porcentual entre la oferta y la demanda hídrica de una zona específica $l_{e}(\%)$, como se indica en la Ecuación 1.

Tabla 1. Categorías del Índice de escasez.

\begin{tabular}{|c|c|c|}
\hline Categoría & Rango (\%) & Interpretación \\
\hline Alto & $>50$ & Alta \\
\hline Medio alto & $21-50$ & Apreciable \\
\hline Medio & $11-20$ & Baja \\
\hline Mínimo & $1-10$ & Muy baja \\
\hline No significativo & $<1$ & No significativa \\
\hline
\end{tabular}

Fuente: Tomado de [29].

$$
I_{e}(\%)=\frac{\text { Demanda hídrica }}{\text { Oferta hídrica }} * 100
$$

Eficiencia del tratamiento en $\mathrm{DBO}_{5}$; para determinar el rango de la eficiencia del tratamiento, se analizan los resultados de los parámetros físico-químicos y microbiológicos del efluente, determinados por el laboratorio de aguas Asebiol SAS, y de esta manera escalar y etiquetar la variable de forma cualitativa (Tabla 2).

Tabla 2. Categorías de la eficiencia de tratamiento en $\mathrm{DBO}_{5}$.

\begin{tabular}{|c|c|c|}
\hline Categoría & Rango (\%) & Interpretación \\
\hline Muy buena & $>80$ & Alta \\
\hline Buena & $60-80$ & Apreciable \\
\hline Regular & $40-59$ & Baja \\
\hline Mala & $20-39$ & Muy baja \\
\hline Deficiente & $<20$ & No significativa \\
\hline
\end{tabular}

Fuente: Elaboración propia.

Minimizar la eficiencia de tratamiento del sistema a un solo parámetro de calidad de 
agua $\left(\mathrm{DBO}_{5}\right)$ es un poco simple, teniendo en cuenta la gran cantidad de indicadores de calidad existentes. Sin embargo, es necesario aclarar que se asumió esta hipótesis dado el incremento significativo de la complejidad del sistema cuando se incluyeron otras variables de calidad. Esta complejidad se agudiza en el momento del consenso de los expertos (debido a una amplitud del espectro de posibilidades y reglas lógicas). De igual forma, se formuló un sistema lo más simple posible en términos de variables de entrada (dada la escasez de la información de calidad del agua y el costo económico que esto conlleva). Finalmente, se asumió que la $\mathrm{DBO}_{5}$ era la variable más crítica de los análisis de calidad del agua y la que más aportaba información al sistema difuso.

Reutilización del agua residual: esta es la variable de salida del sistema, representa el potencial de reutilización de agua residual en términos porcentuales (Tabla 3). Las posibles respuestas son las etiquetas de la variable, las cuales tienen en cuenta las variables de entrada y las reglas basadas en conocimiento experto.

Tabla 3. Categorías de evaluación de la reutilización de aguas residuales.

\begin{tabular}{|c|c|}
\hline Etiqueta & Rango (\%) \\
\hline Reutilización confiable justificada & $80-100$ \\
\hline Reutilización confiable injustificada & $60-80$ \\
\hline Reutilización no confiable justificada & $40-60$ \\
\hline Reutilización no confiable injustificada & $20-40$ \\
\hline No reutilizar & $0-20$ \\
\hline
\end{tabular}

Fuente: Elaboración propia.

Finalmente, las reglas del sistema se adquirieron a través de encuestas dirigidas a expertos en temas como: reutilización de aguas residuales, tratamiento de aguas residuales, microbiología de suelos y fisiología vegetal. Las encuestas lograron identificar las opiniones de los expertos frente a diferentes escenarios. Luego las encuestas fueron sistematizadas y ponderadas para realizar un consenso en la variable de respuesta.

\section{RESULTADOS Y DISCUSIÓN}

\subsection{Balance hídrico}

La Figura 2 representa la hidroclimatología del municipio de Nátaga, la cual corresponde al patrón típico de los Andes. Para el país existen dos estaciones: verano (temporada de menos Iluvia) e invierno (temporada de más lluvia), que están asociadas con el desplazamiento hacia el norte y sur del país de la zona de confluencia intertropical (ZCI) en el año [30]. El patrón de lluvias está caracterizado por dos períodos de lluvias moderados y un estiaje prolongado. La primera temporada de lluvias comienza en enero y se extiende hasta abril, los valores medios mensuales de lluvias oscilan entre 195-230 mm. El segundo período de lluvias se presenta de octubre a diciembre, con valores medios mensuales entre 198 y 235 mm.

Contrario con los resultados encontrados por [31] en el norte del departamento del Huila, los períodos de lluvia del municipio de Nátaga son de similar intensidad y prolongación, lo que se puede explicar por la cercanía del municipio a la Bota Caucana. De igual forma, se presenta solo un periodo de estiaje desde mayo hasta agosto, con valores de lluvias entre $50-120 \mathrm{~mm}$. El promedio de lluvia multianual corresponde a $2.104 \mathrm{~mm}$, el cual corresponde a un clima medio húmedo; sin embargo, el verano es muy prolongado, alrededor de seis meses, y el mes más seco es agosto, con 50 mm. 


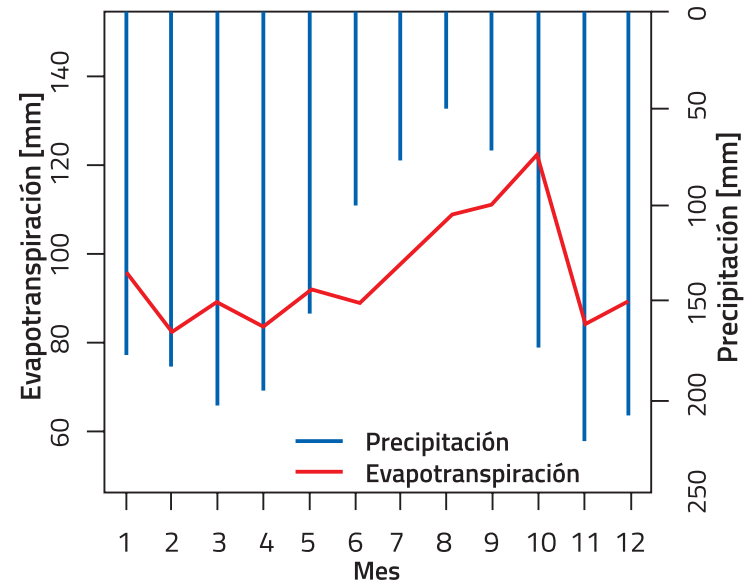

Figura 2. Balance hídrico del municipio de Nátaga, Huila, Colombia.

Fuente: Elaboración propia.

De acuerdo con [30] los dos períodos de lluvia presentados en la Figura 2 se pueden explicar desde la Zona de Convergencia Intertropical (ZCI). En otras palabras, en el trimestre marzo-abril-mayo el eje de la $\mathrm{ZCl}$ está sobre el centro del país, lo que causa la primera temporada invernal del año, mientras que en los meses de mitad del año (junio-julio-agosto) sube hasta la costa Caribe colombiana. De igual forma, desde agosto la ZCI comienza a bajar hacia el centro del país, instalándose allí nuevamente su eje desde septiembre y hasta noviembre, lo que produce la segunda temporada de lluvias.

Por otro lado, es interesante analizar la relación mostrada en la Figura 2 entre la evapotranspiración y la precipitación. Claramente se observa que los meses de mayor evapotranspiración corresponden a los de mayor precipitación, y viceversa. Este resultado pone en evidencia la teoría de [32], la cual establece que el balance hídrico depende de la disponibilidad de agua y de la demanda atmosférica; este modelo representa el suelo como un tanque que almacena agua, la cual es alimentada por la lluvia y eliminada por la evapotranspiración potencial; en este sentido, a medida que no se presente la lluvia tampoco puede incrementarse la evapotranspiración. Es decir, simplifica el concepto de balance a una relación demanda/suministro [33].

\subsection{Implementación del sistema}

\subsubsection{Propuesta metodológica}

Partiendo de la propuesta metodológica desarrollada por [5], se decidió añadir el componente de lógica difusa con el fin de apoyar la toma de decisiones en proyectos de reutilización de aguas residuales tratadas (Figura 3). Este esquema conceptual es general y se puede aplicar a cualquier proyecto de reutilización de aguas residuales.

La Figura 3 muestra un esquema de trabajo que pretende apoyar a las entidades territoriales tomando decisiones basadas en información y conocimiento a través de un sistema experto soportado por lógica difusa. La herramienta en ningún momento pretende reemplazar a los tomadores de decisiones; por el contrario, busca incorporar un criterio más para tomar la mejor decisión.

\subsubsection{Diseño del sistema de lógica difusa}

En la Figura 4 se muestra el Sistema de Inferencia Difusa, el cual consta de las variables de entrada y salida del sistema y la función de membresía. Posteriormente se definió el rango y los subrangos de cada una de las etiquetas lingüísticas fijadas anteriormente para incluir en el sistema las reglas, con el fin de darle funcionamiento y relacionar las 


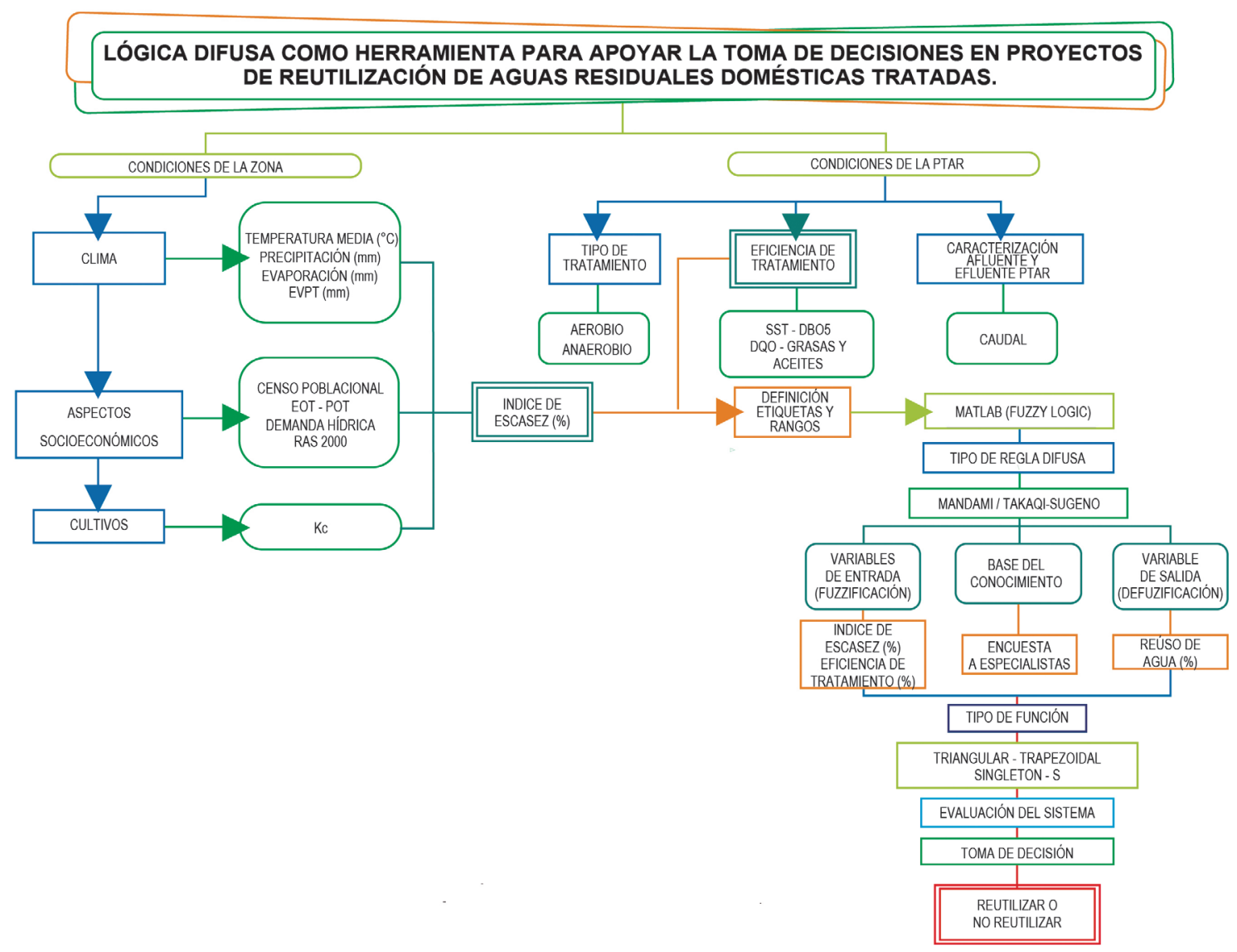

Figura 3. Propuesta metodológica para proyectos de reutilización de aguas residuales.

Fuente: Elaboración propia.

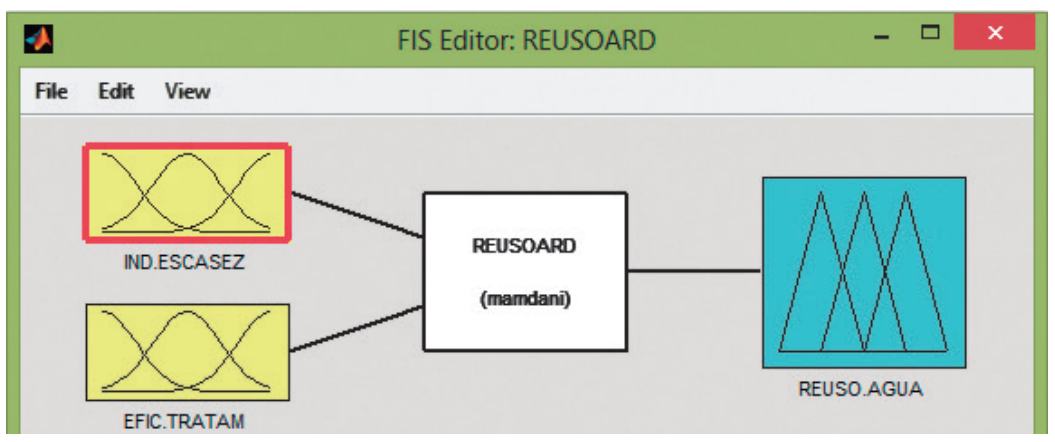

Figura 4. Sistema de Inferencia Difusa.

Fuente: Elaboración propia. 
variables de entrada con la encuesta realizada a los expertos.

Para cada una de las variables de entrada y de salida se definió el rango de manera numérica y la cantidad de subrangos de manera alfanumérica. El tipo de función de membresía que mejor se ajustó a los datos fue la tipo triangular, contrario a lo encontrado por [3], el cual recomienda la función tipo 'Gausiana'. Sin embargo, [3] sugiere que la selección del tipo de función está basada en la información que alimenta al sistema.

\subsubsection{Evaluación del sistema experto en el caso de estudio}

Se evaluó el sistema experto con los datos del caso de estudio (PTAR municipio de Nátaga). Las variables de entrada para la alimentación del sistema fueron un índice de escasez de $28,5 \%$ y eficiencia de tratamiento de $88,7 \%$; como resultado se obtuvo un $92,2 \%$ para la reutilización de agua, que corresponde a una reutilizacion confiable justificada. Este porcentaje generado por el sistema difuso se debe a que el índice de escasez es medio alto, lo que indica que la zona presenta un déficit de agua considerable para contemplar una reutilización con algún fin específico (Tabla 4).

La evaluación del sistema evidencia cómo la eficiencia del tratamiento representa la confiabilidad del sistema, principalmente en términos de calidad del agua, y el Índice de escasez representa la justificación del sistema en términos de la necesidad del recurso hídrico (cantidad).

La Figura 5 analiza la influencia de la variable de entrada en la superficie de respuesta. En este caso de estudio se evidencia la importancia de la eficiencia de la PTAR en la respuesta del sistema: esto se puede explicar porque la condición climática no es tan crítica (clima medio húmedo). Sin embargo, en otras condiciones ambientales con problemas de sequía prolongadas, el entendimiento de la dinámica hídrica representada en el Índice de escasez es fundamental para establecer estrategias locales de adaptación frente al cambio climático.

Otros modelos de inferencia pueden ser utilizados para resolver problemas complejos, como la identificación de fuentes alternas de recurso hídrico. Por ejemplo, las redes bayesianas propuestas por investigadores como [34], serían formas alternativas de inferir las reglas del sistema difuso. Esta metodología podría ser una forma interesante de comparar resultados.

Sin embargo, la herramienta hidroinformática propuesta es de mucha aplicación en el sector de saneamiento básico rural, especialmente

Tabla 4. Evaluación del sistema experto basado en lógica difusa.

\begin{tabular}{|c|c|c|c|}
\hline \multicolumn{2}{|c|}{ Variables del sistema } & Caso de estudio & Reglas del sistema \\
\hline \multirow{2}{*}{ Entrada } & Índice de escasez $(\%)$ & 28,5 & \multirow{2}{*}{$\begin{array}{c}\text { Encuesta a expertos } \\
\text { y ponderación }\end{array}$} \\
\cline { 2 - 3 } & Eficiencia de tratamiento $(\%)$ & 88,7 & \\
\hline Salida & Reutilización de agua $(\%)$ & 92,2 & \\
\hline
\end{tabular}

Fuente: Elaboración propia. 


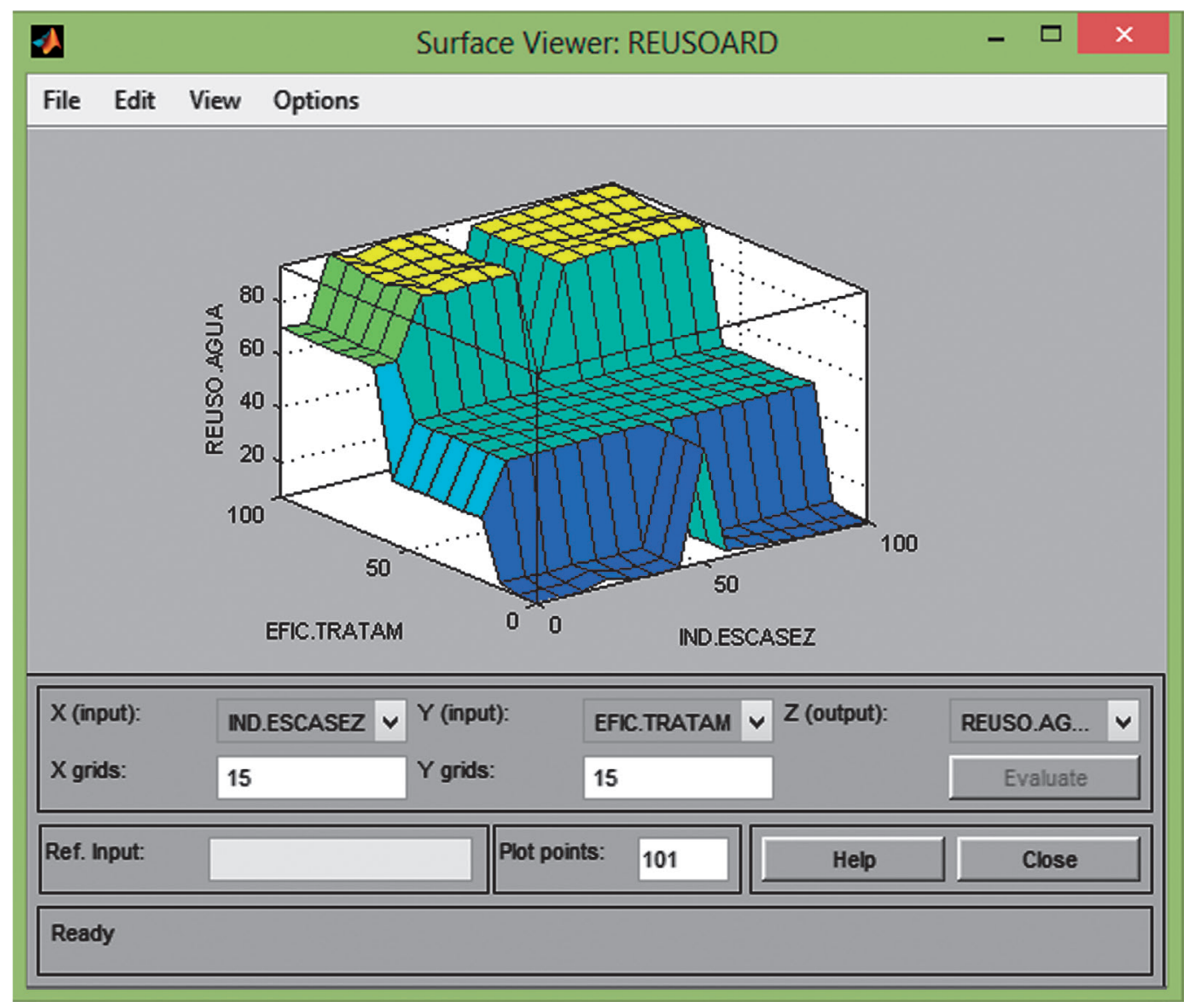

Figura 5. Superficie de respuesta del sistema en 3D.

Fuente: Elaboración propia.

para secretarios de medio ambiente o desarrollo rural, los cuales deben viabilizar proyectos de reutilización de aguas residuales tratadas. En este sentido, la herramienta pretende apoyar al personal técnico para que tome una decisión más acertada teniendo en cuenta el conocimiento experto y la información disponible de la zona. Además, este sistema está hecho en una interfaz de fácil entendimiento y manipulación, en donde solo basta digitar los valores de cada una de las variables para obtener un resultado.

\section{CONCLUSIONES}

Se demuestra que, en regiones con problemas de sequías prolongadas, el desarrollo de herramientas hidroinformáticas es de mucha utilidad para los tomadores de decisiones al momento de identificar o reactivar fuentes alternas de recursos hídricos que suplan la demanda de la población. La herramienta que se diseñó para estimar el potencial de reutilización de aguas residuales tratadas a partir de variales de entrada, como el Índice 
de escasez y la eficiencia de tratamiento en $\mathrm{DBO}_{5}$ de una PTAR fue implementada en las condiciones ambientales del municipio de Nátaga. Los resultados muestran que la eficiencia del tratamiento en $\mathrm{DBO}_{5}$ es $88,7 \%$; es decir, que el tratamiento que se realiza en la PTAR al afluente es confiable. De igual forma, el Índice de escasez es de 28,5, que correspondea un índice medio alto, lo que indica que la oferta hídrica no suple completamente la demanda de la población teniendo en cuenta los factores hidrológicos para el cálculo de la oferta de agua superficial. Finalmente, la evaluación del sistema experto basado en lógica difusa concluye que la reutilización de agua para el municipio de Nátaga, Huila, se encuentra en el rango de reutilización confiable justificada, con un $92,2 \%$.

\section{AGRADECIMIENTOS}

Especial agradecimiento a la Alcaldía del municipio de Natagá por el acceso a la información de la PTAR. De igual forma, al comité de expertos que decidieron aportar su experiencia y conocimiento para alimentar las reglas del sistema difuso: Eduardo Valencia (Usco), Jaime A. Lara (PUJ) y Norberto Urrutia Cobo (Univalle). También a los revisores anónimos, quienes aportaron para mejorar la calidad del manuscrito.

\section{BIBLIOGRAFÍA}

[1] Akdeniz, E., \& Bagriyanik, M. (2016). A knowledge based decision support algorithm for power transmission system vulnerability impact reduction. International Journal of Electrical Power \& Energy Systems, 78, pp. 436-444. doi: 10.1016/j.ijepes.2015.11.041
[2] Murla, D., Gutiérrez, O., Martínez, M., Suñer, D., Malgrat, P., \& Poch, M. (2016). Coordinated management of combined sewer overflows by means of environmental decision support systems. Science of the Total Environment, 550, pp. 256-264. doi: 10.1016/j. scitotenv.2016.01.076

[3] Almeida, G., Vieira, J., Marques, A., Kiperstok, A., \& Cardoso, A. (2013). Estimating the potential water reuse based on fuzzy reasoning. Journal of Environmental Management, 128, pp. 883-892. doi: 10.1016/j. jenvman.2013.06.048

[4] Valencia, E., Aragón, R., \& Romero, J. (2012). Potencial de reutilización del efluente de la planta de tratamiento de aguas residuales de Nátaga en cultivo de cacao (Theobroma cacao L.). Revista UDCA. Actualidad \& Divulgación Científica, 15, pp. 77-86.

[5] Valencia, E., Aragón, R., Romero, J. (2010). Esquema metodológico para reutilización de aguas residuales domésticas tratadas en riego. Revista Ingeniería de Recursos Naturales y del Ambiente, 9, pp. 55-60.

[6] Food and Agriculture Organization of the United Nations FAO. (1999). Wastewater Treatment and Use in Agriculture. En: http://www.fao.org/docrep/T0551E/ t0551e04.htm

[7] Organización Mundial de la Salud (OMS). (2006). Guidelines for the safe use of wastewater, excreta and greywater. Volume 2: Wastewater use in agriculture. En: http:/l www.who.int/water_sanitation_health/ wastewater/gsuweg2/en/index.html 
[8] MADS (2015). Decreto 3930 de 2010. Norma de vertimientos puntuales a cuerpos de aguas superficiales y a los sistemas de alcantarillado público. En: http://www. alcaldiabogota.gov.co/sisjur/normas/ Norma1.jsp?i=40620

[9] Silva, J., Torres, P., \& Madera, C. (2008). Reúso de aguas residuales domésticas en agricultura. Una revisión. Agronomía Colombiana, 26(2), pp. 347-359.

[10] Minagricultura(2011). Proyecto: Desarrollo de capacidades en el uso seguro de aguas residuales para agricultura. En: http://www. ais.unwater.org/ais/pluginfile.php/356/ mod_page/content/128/Colombia_ Informe\%20Nacional.pdf

[11] Ideam (2015). Estudio Nacional del Agua 2014. Bogotá, D.C., pp. 496.

[12] Lasso, J., \& Ramírez, J. L. (2011). Perspectivas generales del efecto del reúso de aguas residuales para riego en cultivos para la producción de biocombustibles en Colombia. El hombre y la máquina, 36, pp. 95-105.

[13] Yi, L., Jiao, W., Chen, X., \& Chen, W. (2011). An overview of reclaimed water reuse in China. Journal of Environmental Sciences, 23 (10), pp. 1585-1593. doi:10.1016/ S1001-0742(10)60627-4

[14] CAM (2013). Plan de Acción 2012-2015: Territorio Verde y climáticamente inteligente. Corporación Autónoma Regional del Alto Magdalena (CAM). Neiva, Huila, p. 180.

[15] WWAP (United Nations World Water Assessment Programme). (2015). The United Nations World Water Development
Report 2015: Water for a Sustainable World. Paris, Unesco, pp. 77.

[16] Ruiz, J. (2010). Cambio climático en temperatura, precipitación y humedad relativa para Colombia usando modelos meteorológicos de alta resolución. Panorama 2011-2100. Nota técnica 005/2010 del Ideam.

[17] Huang, Y., Lan, Y., Thomson, S., Fang, A., Hoffmann, W., \& Lacey, R. (2010). Development of soft computing and applications in agricultural and biological engineering. Computers and Electronics in Agriculture, 71, pp. 107-127. doi:10.1016/j.compag.2010.01.001

[18] Obregón, N., \& Romero, J. (2013). Aplicaciones de sistemas inteligentes en Ingeniería Agrícola. Neiva, Huila: Editorial Universidad Surcolombiana, pp. 163.

[19] Tkach, R. J., \& Simonovic, S. P. (1997). A new approach to multi-criteria decision making in water resources. Journal of Geographic Information and Decision Analysis, 1 (1), pp. 25-43.

[20] Hayes, M. J. (1999). Drought Indices. National Drought Mitigation Center. En: http://drought.unl.edu/

[21] Zarghami, M., Abrishamchi, A., \& Ardakanian, R. (2007). Multi-criteria decision making for integrated urban water management. Water Resources Management, 22 (8), pp. 1017-1029. doi: $10.1007 / 511269-007-9207-7$

[22] Villegas, P. A., Obregón, N., Lara, J. A., Méndez, S., \& Luna, A. (2009). Herramienta informática como apoyo 
en la toma de decisiones en proyectos de agua y saneamiento en comunidades indígenas. Avances en Recursos Hidráulicos, 19, pp. 39-54.

[23] Souza, A. L., Matsura, E. E., \& Miranda, J. H. (2005). Teoría de conjuntos fuzzy aplicada al estudio de la dinámica del agua y de los solutos en el suelo. Revista Estudios de la zona no saturada del suelo, 7, pp. 275-279.

[24] Méndez, Z., Villegas, P. (2005). Los sistemas difusos como herramienta de modelación de la producción frutícola, En: Día Matlab, Univerisidad del Bosque. Bogotá, D.C., Colombia.

[25] Bohórquez, P. A., Leiva, L., \& Noscue, F. (2007). Esquema de Ordenamiento Territorial, municipio de Nátaga, Huila.

[26] Unesco-IMTA (2006). Evaluación de recursos hídricos. Elaboración de balances hídricos por cuencas hidrográficas. Instituto Mexicano de Tecnología del Agua. México.

[27] Salazar, L. A. (2012). Diseño e implementación de un controlador difuso en un dsPIC30F4011 para una hélice de paso variable (memoria de estadía profesional). Facultad de Ingeniería, Departamento de Ingeniería Mecatrónica, Universidad Politécnica de Pachuca, Pachuca.

[28] Zadeh, L. A. (1965). Fuzzy sets. En: Information and Control, 8, pp. 338-353. doi: 10.1016/s0019-9958(65)90241-x

[29] MAVDT (2004). Grupo de Análisis Económico. Metodología para el cálculo del índice de escasez. Bogotá, D.C., Colombia.
[30] Henríquez, M. (2012). Climatología ambiental de Colombia. Una guía sobre la relación clima-ambiente para los estudiantes y profesionales de las carreras ambientales. Bogotá, D.C., Colombia: Universidad Santo Tomás, pp. 330.

[31] Romero, J., Chavarro, J., Garrido, A., \& Guzmán, H. (2014). Performance evaluation of irrigation techniques through the implementation of a fuzzy logic system. ARPN Journal of Engineering and Applied Sciences, 9(7), pp. 1087-1093.

[32] Budyko, M. I. (1958). The Heat Balance of the Earth's Surface. US Department of Commerce, Washington, DC. USA.

[33] Zhang, L., Potter, N., Hickel, K., Zhang, Y., \& Shao, Q. (2008). Water balance modeling over variable time scales based on the Budyko framework. Model development and testing. Journal of Hydrology. 360, pp. 117-131. doi:10.1016/j. jhydrol.2008.07.021

[34] Kjréulff, U. B., \& Madsen, A. L. (2013). Bayesian Networks and Influence Diagrams: a Guide to Construction and Analysis. Springer, USA, pp. 382. doi: 10.1007/9781-4614-5104-4 